\title{
Tamanho de amostra para avaliar a densidade populacional de lagartas em lavouras de soja
}

\author{
Sample size to assess caterpillars population density in soybean field \\ Claudia Carolina Cabral Antúnez ${ }^{1 *}$, Lindolfo Storck ${ }^{2}$, Jerson Vanderlei Carús Guedes ${ }^{2}$, \\ Jimmy Walter Rasche Alvarez ${ }^{1}$, Gilmar Luiz Schaefer ${ }^{2}$ e Alberto Cargnelutti Filho ${ }^{2}$
}

\author{
${ }^{1}$ Universidad Nacional de Asunción, Facultad de Ciencias Agrarias, Ruta Mariscal Estigarribia Km 10,5, Campus \\ Universitario, San Lorenzo, Paraguay. \\ 2 Universidade Federal de Santa Maria, Departamento de Defesa Fitossanitária, Av. Roraima, n 1.000, Camobi, CEP \\ 97105-900 Santa Maria, RS, Brasil. \\ * Autor para correspondencia (claudia_c_cabral@hotmail.com) \\ Recibido: 01/06/2016; Aceptado: 14/11/2016. \\ 10.18004/investig.agrar.2016.dicie mbre.70-76
}

\section{RESUMO}

A amostragem de pragas é uma das bases para a toma de decisão no manejo de pragas em lavouras de soja. Assim, determinar o plano amostral mais adequado consiste em definir a unidade amostral, o método de amostragem e o tamanho de amostra para a quantificação da entomofauna. O objetivo foi estimar o tamanho de amostra para medir a densidade populacional de espécies de lagartas, variando métodos de coleta, altitude e cultivares de soja. Foram amostradas 100 lavouras de soja, distribuídas em nove municípios da região central do Rio Grande do Sul, em três safras agrícolas (2010/2011, 2011/2012, 2012/2013). Em cada lavoura foram demarcados 30 pontos distanciados 20 metros entre si. Em cada ponto foram coletadas lagartas das espécies Chrysodeixis includens, Anticarsia gemmatalis e Rachiplusia $n u$, por meio dos métodos pano-de-batida largo e rede entomológica, totalizando 6.000 coletas. Para cada lavoura, método de coleta e espécie de lagarta foi estimado a média da densidade populacional e o tamanho de amostra por meio de reamostragem. Usando o pano-de-batida largo para a coleta de lagartas em soja, para um erro de estimação (amplitude do intervalo de confiança) igual a dois lagartas e densidade populacional na classe $>2$ lagartas. $\mathrm{m}^{-2}$, o tamanho de amostra é 47, 78 e 18, respectivamente, para as espécies $C$. includens, A. gemmatalis e $R$. nu e para rede o tamanho de amostra é 19 e 43, respectivamente, para as espécies $C$. includens $e$ A. gemmatalis. Os resultados indicam que o tamanho de amostra (número de pontos) para determinar a densidade populacional de lagartas varia com o método de coleta, altitude, cultivar e a densidade populacional.

Palavras-chave: Glycine $\max$ amostragem, reamostragem, dimensionamento amostral.

\begin{abstract}
Sampling is one of the basis for decision-making to control pests in soybean crops. Thus, to determine the most appropriate sampling plan is to define the sampling unit, the sampling method and sample size for the quantification of insect population. The objective of the study was to estimate the sample size to measure caterpillar species population density, with varying methods of collection, of altitude and of soybean cultivars. Samples were taken from 100 soybean fields, located in nine districts in the central region of Rio Grande do Sul, during three growing seasons (2010/2011, 2011/2012, 2012/2013). In each field, 30 points separated by 20 meters each were marked off. At each point caterpillars of the species Chrysodeixis includens, Anticarsia gemmatalis and Rachiplusia nu were collected through the wide-beating cloth-methods and entomological network, totaling 6,000 units. The sample size (number of points) to determine the density of tracks varies with the method of collection and population density. Using the wide-beating cloth method for soybean caterpillars collection for an estimation error (confidence interval amplitude) equal to two caterpillars and population density in the class $>2$ caterpillars. $\mathrm{m}^{-2}$, the sample size is 47,78 and 18 , respectively, for the $C$. includens, A. gemmatalis and Rachiplusia nu species, as for the entomological network method, sample sizes are 19 and 43 for the $C$. includens e A. gemmatalis species. The study shows that sample size (number of points) to determine caterpillars population density varies according to, collection method, altitude, soybean cultivars and population density.
\end{abstract}

Key words: Glycine max, sampling, resampling, sample dimension. 


\section{INTRODUÇÃO}

Nos últimos 50 anos houve aumento no interesse dos produtores pela cultura da soja, devido principalmente a explosão do preço no mercado mundial, isso fez com que a área cultivada no Brasil crescesse de 240 mil ha em 1961 a 31,5 milhões de ha em 2014/2015 (CONAB 2015). A produtividade de grãos de soja está relacionada com a ocorrência de insetos-pragas que causam perdas e aumentam os custos de produção.

Entre as principais pragas da soja estão as lagartas das espécies Anticarsia gemmatalis (Hübner 1818), Chrysodeixis (=Pseudoplusia) includens (Walker 1857) e Rachiplusia nu (Guenée 1852), que são de hábito filófago, de grande distribuição no Brasil, desde Rio Grande do Sul até os estados da região centro-oeste (Guedes et al. 2011). Vários autores citam que no Estado de Rio Grande do Sul suas populações têm aumentado nos últimos anos (Guedes et al. 2011, Alexandre 2010). Esses autores comentam a existência de nova dinâmica em relação a alterações na época de ocorrência, na intensidade e proporção das espécies.

Considerando que a ocorrência de insetos-pragas pode causar grandes perdas nas culturas, é necessário realizar amostragem para conhecer a presença e a distribuição das lagartas nas suas diferentes fases do ciclo biológico para poder controla-los adequadamente. Assim, é importante monitorar as lavouras quanto à densidade populacional desses insetos durante o estágio de desenvolvimento da cultura.

Os programas de manejo integrado de pragas (MIP) se baseiam em amostragem de pragas e para implementar o MIP são necessários conhecer o método de coleta e o tamanho de amostra, que permitam estimar a densidade populacional da praga com erro de estimação conhecido. Métodos de coleta por pano-de-batida, pano vertical, rede entomológica e contagem absoluta podem ser usados para a estimativa da densidade populacional de pragas de soja. O pano-de-batida largo é um dos métodos de coleta mais recomendado (Corrêa-Ferreira 2012, Stürmer et al. 2012). Por outro lado, a rede entomológica é o método mais usado para coleta de insetos quando há presença de maior número de ordens ou de insetos com maior agilidade (Lara et al. 2008).

Nas lavouras de soja são realizadas amostragens para quantificar a presença de insetos pragas. No entanto, a definição da unidade amostral, do método de amostragem e do número de amostras varia amplamente, motivo pelo qual existe necessidade da definição de umplano amostral adequado para as condições de Rio Grande do Sul. Na cultura de soja, há indicações de tamanho de amostra diferentes, assim na literatura são relatados diferentes tamanhos de amostras, oito pontos amostrais (Gallo et al. 2002), dez pontos (Corrêa-Ferreira 2012), 18 pontos para um erro de estimação (semiamplitude do intervalo de confiança; $1-\mathrm{p}=0,95)$ igual a $30 \%$ da média estimada, para determinar a densidade populacional de lagartas, numa lavoura de 6,1 ha, nos estágios fenológicos intermediários da soja (R1, R2, R3, R4 e R5.1) (Stürmer et al. 2013).

Diante da necessidade de informações que possibilitem recomendações de métodos de coleta e respectivo tamanho de amostra para as principais pragas da cultura de soja, em condições ambientais variadas. O objetivo deste trabalho foi determinar o tamanho de amostra para estimar a densidade populacional de algumas espécies de lagartas que atacam a soja, variando o método de coleta, cultivar de soja e altitude da área.

\section{MATERIAL E MÉTODOS}

O estudo foi realizado em 100 lavouras comerciais de soja, mantidas com manejo mínimo de pragas realizado pelo produtor, variando as cultivares e os estágios de desenvolvimento. Os municípios do estado de Rio Grande do Sul onde as lavouras se localizaram foram: São Sepé, Formigueiro, Restinga Seca, Itaara, Júlio de Castilhos, São Pedro do Sul, Dilermando de Aguiar, Santa Maria e São Gabriel, nas safras de 2010/11, 2011/12 e 2012/13, avaliando-se 41, 30 e 29 lavouras, respectivamente.

Em cada lavoura foram demarcados 30 pontos equidistantes em 20 metros, sendo 10 pontos em cada fileira de soja, e as fileiras distanciadas a 20 metros uma da outra. Em cada um dos 30 pontos, foram realizadas as coletas dos insetos presentes, empregando dois métodos de coleta: pano-de-batida largo e rede entomológica, uma em cada lado (esquerdo e direito) dos pontos.

O pano-de-batida largo é um pano branco de 1,0 $\mathrm{m} \mathrm{de}$ comprimento por $1,0 \mathrm{~m}$ de largura, com as bordas inseridas em cabo de madeira (Stürmer et al. 2012). Este pano é colocado desde a base de uma fileira de soja até a outra fileira e as plantas de um lado são batidas vigorosamente sobre o pano, de modo a que os insetos pragas presentes fiquem no pano. Neste caso, a área amostrada em cada ponto é igual a $0,5 \mathrm{~m}^{2}$. A rede 
entomológica é constituída de um cabo de madeira de 1,0 $\mathrm{m}$, com aro de $0,35 \mathrm{~m}$ de diâmetro. As coletas dos insetos com a rede entomológica foram realizadas por meio de cinco passadas da rede sobre as plantas de duas fileiras e um metro linear, seguindo a linha imaginária de um "oito aberto" resultando numa área de $1,0 \mathrm{~m}^{2}$ (Panizzi e CorrêaFerreira 1978).

O número de lagartas das espécies Chrysodeixis includens, Anticarsia gemmatalis e Rachiplusia nu, foi anotado em planilha eletrônica Excel e ajustado para 1,0 $\mathrm{m}^{2}$, com a respectiva identificação da lavoura, ponto amostral com um GPS e método de coleta.

Para cada uma das 100 lavouras, cada método de coleta e cada espécie, foi estimada a média e determinado o tamanho de amostra pelo método de reamostragem (Efron 1979, Ferreira 2005, Storck et al. 2012). Esse procedimento estatístico é adequado, tendo em vista que os valores do número de insetos por ponto (densidade populacional), em geral, não seguem distribuição de probabilidade conhecida ou não, e é a mesma para os diferentes ambientes, método de coleta e espécies (Barbosa 2001). Para isto, usando os valores do número de lagartas, de cada espécie, obtidos nos 30 pontos da lavoura, foram geradas 2.000 reamostras (J), com reposição, e calculada a média de cada reamostra. Estas 2.000 estimativas de médias, foram ordenadas para a identificação do quantil 0,025 como limite inferior (LI) e do quantil 0,975 como limite superior (LS). Os valores de LI e LS correspondem à estimativa do intervalo de confiança (IC) com taxa de erro igual a 0,05. O mesmo procedimento, 2.000 reamostras com reposição, foram repetidos para os tamanhos de amostra igual a $\mathrm{k}(\mathrm{k}=3,4$, ..., 2000) em cada lavoura.

Para cada valor de k, as 2.000 médias, obtidas com as 2.000 reamostras de tamanho $\mathrm{k}$, foram ordenadas para a identificação dos $\mathrm{LI}(\mathrm{k})$ e $\mathrm{LS}(\mathrm{k})$. Os limites $\mathrm{LI}(\mathrm{k})$ e $\mathrm{LS}(\mathrm{k})$ definem o intervalo de confiança, com taxa de erro igual a 0,05 , para $\mathrm{k}$ pontos amostrados por lavoura. A amplitude do intervalo de confiança (AIC), para amostra de tamanho $\mathrm{k}$, é estimado por $\mathrm{AIC}(\mathrm{k})=\mathrm{LS}(\mathrm{k})-\mathrm{LI}(\mathrm{k})$. O valor de $\mathrm{k}$, para o qual a $\mathrm{AIC}(\mathrm{k})$ é igual ao AIC previamente admitido, é o tamanho de amostra (número de pontos por lavoura), com 1-p =0,95 de confiança. Para estes procedimentos estatísticos foi usado o software $\mathrm{R}$ ( $\mathrm{R}$ DEVELOPMENT CORE TEAM 2014).

As densidades populacionais das espécies, para as coletas com pano-de-batida largo e rede entomológica, para cada cultivar e altitude, foram comparadas pelo teste $\mathrm{t}$ por reamostragem, usando o aplicativo BioEstat 5.3 (Ayres et al. 2007). As demais análises estatísticas foram realizadas com o aplicativo Microsoft Office Excel.

\section{RESULTADOS E DISCUSSÃO}

Nas 100 lavouras estudadas foi constatado 16 cultivares de soja, das quais as oito mais frequentes estão listados na Tabela 1. As cultivares BMX Potencia RR (25\%) e Nidera 5009 (20\%) estiveram presentes em 45\% das lavouras nas três safras. Em 39\% das lavouras foram constatadas altitudes acima de $150 \mathrm{~m}$ (Tabela 2), correspondendo a três municípios (Itaara, Júlio de Castilhos e São Sepé). A altitude dos locais variou entre 55 e $494 \mathrm{~m}$, a latitude entre $29^{\circ} 15^{\prime}$ e $30^{\circ} 19^{\prime}$ e a longitude entre $53^{\circ} 50^{\prime}$ e $54^{\circ} 28^{\prime}$. Das lavouras avaliadas, $55 \%$ se encontravam no estágio vegetativo.

As densidades populacionais referentes as espécies $C$. includens e $R$. $n u$ e dois métodos de coleta foram maiores nas lavouras de menor altitude (altitude $<150 \mathrm{~m}$ ), e densidade populacional de A. gemmatalis não foi influenciada pela altitude nos dois métodos de coleta. Esse fato poderia ser explicado, em parte, pelos fatores abióticos como a temperatura e umidade relativa do ar que poderia afetar a abundância destes insetos, pois em ambientes mais úmidos e frescos há maior possibilidade de reprodução de fungos como Nomuraea rileyi e virus como Baculovirus anticarsia (Ignoffo 1992, Cespedes et al. 2008, Sujii et al. 2002, Panizzi e Silva 2009, Gutierrez 2009).

A densidade populacional das três espécies de lagartas ( $C$. includens; A. gemmatalis e $R$. nu), não diferiu na maioria dos cultivares (Tabela 1), para os dois métodos de coleta, com exceção de $C$. includens coletada em menor número pela rede no cultivar BMX TURBO RR e A. gemmatalis e $R$. $n u$ que foram menores pelo método de rede no cultivar CODETEC 231, isto poderia ser explicado por uma possível diferença na composição química dos cultivares assim como diferenças morfológicas que poderiam existir, tornando que o alimento seja ou não preferido. É importante recordar que o alimento é um dos fatores mais importantes, influindo diretamente sobre a distribuição e abundancia de insetos (Franco et al. 2014).

Nas cultivares NIDERA 5009, A8000, Tijereta LT 2162, BMX TURBO RR e NIDERA 6411 se registraram densidade de $C$. includens menor do que o limite inferior do intervalo de confiança, para os dois métodos de coleta. 
Nas cultivares NIDERA 5009, A8000, Tijereta LT 2162, densidade de $C$. includens menor do que o limite inferior BMX TURBO RR e NIDERA 6411 se registraram do intervalo de confiança, para os dois métodos de coleta.

Tabela 1. Frequência de lavouras (Fo), média (número de lagartas $\mathrm{m}^{-2}$ ), com coleta por pano-de-batida largo e por rede entomológica, limite inferior (LI) e superior (LS) do intervalo de confiança por reamostragem $(1-\mathrm{p}=0,95)$.

\begin{tabular}{|c|c|c|c|c|c|c|c|}
\hline \multirow[t]{2}{*}{ Cultivar } & \multirow{2}{*}{$\begin{array}{l}\text { Fo } \\
(\%)\end{array}$} & \multicolumn{2}{|c|}{ C. includens } & \multicolumn{2}{|c|}{ A. gemmatalis } & \multicolumn{2}{|c|}{ R. $n u$} \\
\hline & & Pano & Rede & Pano & Rede & Pano & Rede \\
\hline BMX POTENCIA RR & 25 & $2,93 \mathrm{a}$ & $0,73 \mathrm{~A}$ & $0,97 \mathrm{~b}$ & $0,25 \mathrm{~A}$ & $1,08 \mathrm{~b}$ & $0,22 \mathrm{~A}$ \\
\hline NIDERA 5009 & 20 & $0,99 \mathrm{a}$ & $0,14 \mathrm{~A}$ & $1,62 \mathrm{a}$ & $0,48 \mathrm{~A}$ & $0,38 \mathrm{a}$ & $0,09 \mathrm{~A}$ \\
\hline CODETEC 231 & 10 & $2,99 \mathrm{a}$ & $0,58 \mathrm{~A}$ & $1,81 \mathrm{ab}$ & $0,57 \mathrm{~B}$ & $0,72 \mathrm{~b}$ & $0,22 \mathrm{~B}$ \\
\hline A 8000 & 10 & $0,78 \mathrm{a}$ & $0,25 \mathrm{~A}$ & $1,99 \mathrm{a}$ & $1,50 \mathrm{~A}$ & $1,08 \mathrm{a}$ & $0,07 \mathrm{~A}$ \\
\hline TIJERETA LT 2162 & 6 & $0,19 \mathrm{a}$ & $0,03 \mathrm{~A}$ & $0,91 \mathrm{a}$ & $0,21 \mathrm{~A}$ & $0,04 \mathrm{a}$ & $0,00 \mathrm{~A}$ \\
\hline CODETEC 5909 & 6 & $2,48 \mathrm{a}$ & $0,69 \mathrm{~A}$ & $0,35 \mathrm{a}$ & $0,17 \mathrm{~A}$ & $0,89 \mathrm{a}$ & $0,12 \mathrm{~A}$ \\
\hline BMX TURBO RR & 6 & $0,72 \mathrm{a}$ & $0,15 \mathrm{~B}$ & $0,19 \mathrm{a}$ & $0,01 \mathrm{~A}$ & $0,51 \mathrm{a}$ & $0,00 \mathrm{~A}$ \\
\hline NIDERA 6411 & 5 & $0,59 \mathrm{a}$ & $0,25 \mathrm{~A}$ & $1,07 \mathrm{a}$ & $0,91 \mathrm{~A}$ & $0,28 \mathrm{a}$ & $0,08 \mathrm{~A}$ \\
\hline Média & - & 1.695 & 0.404 & 1.158 & 0.440 & 0.680 & 0.142 \\
\hline LI & - & 1.229 & 0.260 & 0.701 & 0.226 & 0.459 & 0.100 \\
\hline LS & - & 2.116 & 0.554 & 1.584 & 0.686 & 0.902 & 0.183 \\
\hline
\end{tabular}

Médias seguidas de letra diferente na linha, minúscula para pano e maiúscula para rede, diferem significativamente pelo teste t (p <0,05; 4.000 reamostras).

Para as cultivares CODETEC 5909 e BMX TURBO RR registraram-se densidades de A. gemmatalis menores do que o limite inferior do intervalo de confiança, para os dois métodos de coleta e na TIJERETA LT 2162 registrou-se menor número pelo método de rede. Por sua vez, as cultivares NIDERA 5909 e TIJERETA LT 2162 apresentaram densidades de $R$. nu menores do que o limite inferior do intervalo de confiança, para os dois métodos de coleta e nas cultivares A 8000, BMX TURBO RR e NIDERA 6411 houve densidades menores da espécie $R$. nu para o método de rede.
Essas inferioridades estão confundidas com os ambientes (altitude, manejo e outros) e a prática do controle de insetos diferenciados. Valores obtidos no pano-de-batida largo discriminam mais as diferenças entre as cultivares, se comparados com a rede entomológica, possivelmente, por haver maior densidade populacional. A cultivar é um fator importante que deve ser considerado no manejo das pragas na cultura, pois influi diretamente na abundância e distribuição dos insetos (Franco et al. 2014).

Tabela 2. Número médio de lagartas $\mathrm{m}^{-2}$, de acordo com a espécie, o método de coleta e a classe de altitude da lavoura.

\begin{tabular}{lcccl}
\hline \multicolumn{1}{c}{ Espécie de lagarta } & \multicolumn{2}{c}{ Altitude $<150 \mathrm{~m}$} & \multicolumn{2}{c}{ Altitude $>150 \mathrm{~m}$} \\
\hline \multirow{2}{*}{ C. includens } & Pano & Rede & Pano & Rede \\
\cline { 2 - 5 } & $2,23 \mathrm{aA}$ & $0,54 \mathrm{aA}$ & $0,86 \mathrm{abB}$ & $0,19 \mathrm{aB}$ \\
\hline A. gemmatalis & $1,24 \mathrm{bA}$ & $0,38 \mathrm{abA}$ & $1,03 \mathrm{aA}$ & $0,54 \mathrm{aA}$ \\
\hline$R . n u$ & $0,95 \mathrm{bA}$ & $0,17 \mathrm{bA}$ & $0,25 \mathrm{bB}$ & $0,10 \mathrm{aA}$
\end{tabular}

Médias não seguidas pela mesma letra (minúscula na vertical e maiúscula entre altitude dentro de método) diferem pelo teste $\mathrm{t}(\mathrm{p}<0,05 ; 4.000$ reamostras).

Pelas estimativas por intervalo (LI e LS), na média das cultivares, a densidade populacional resultante da coleta por pano-de-batida foi significativamente superior à da rede entomológica para as espécies de lagarta estudadas (Tabela 1). Deve ser lembrado que, a comparação da área ou volume de plantas cobertas pelos dois métodos de coleta é dificultada, pois o resultado da coleta da rede depende da repetitividade dos movimentos do operador e, possivelmente, do horário de coleta, devido a posição dos insetos dentro do dosseldas plantas.

As médias da densidade populacional, por classe de densidade, e o tamanho de amostra para uma amplitude do intervalo de confiança $($ AIC) igual a um $($ AIC $=1)$ e 
dois (AIC = 2) lagartas por ponto, de cada espécie e método de coleta, são apresentadas na Tabela 3 . Considerando as três espécies de lagartas, observa-se frequência média de $26 \%$ das lavouras sem lagartas, pelo método do pano-de-batida largo, e $23 \%$ com o método da rede entomológica. Este fato repercute na baixa estimativa da média da densidade populacional das lagartas. A baixa densidade populacional, também foi relatada por Stürmer et al. (2014) o qual pode ser devido a frequência de controle das pragas praticada pelos produtores.

Para as três espécies e os dois métodos de coleta, com o acréscimo da média de lagartas encontradas (classes de densidade populacional) há aumento do tamanho de amostra. Esta relação se deve a forma de quantificar o erro de estimação (AIC) em número de lagartas. No caso em que essa amplitude é atribuída como sendo uma proporção da média da densidade populacional (Stürmer et al. 2014), esta relação é inversa, ou seja, menores densidades populacionais implicam em maiores tamanhos de amostra.

Considerando-se uma lavoura com classe de densidade populacional superior a 2,00 lagartas $\mathrm{m}^{-2}$ como sendo um valor mais alto e sendo o erro de estimação igual a dois lagartas (AIC=2), o tamanho de amostra é igual a 46,5 para $C$. includens, 77,5 para A. gemmatalis e 54,7 para $R$. $n u$, usando o pano-de-batida como método de coleta (Tabela 3).

Tabela 3. Frequência de lavouras (Fo), média da densidade populacional (lagartas $\mathrm{m}^{-2}$ ) e tamanho de amostra para a amplitude do intervalo de confiança (AIC), de acordo com a espécie, o método de coleta e a classe da densidade populacional.

\begin{tabular}{|c|c|c|c|c|c|c|c|c|}
\hline \multirow[t]{2}{*}{ Classe } & \multicolumn{4}{|c|}{ Pano-de-batida largo } & \multicolumn{4}{|c|}{ Rede entomológica } \\
\hline & Fo & Média & $\mathrm{AIC}=1^{1}$ & $\mathrm{AIC}=2$ & Fo & Média & $\mathrm{AIC}=1$ & $\mathrm{AIC}=2$ \\
\hline & \multicolumn{8}{|c|}{ Chrysodeixis includens } \\
\hline 0 & 11 & 0 & - & - & 23 & 0 & - & - \\
\hline 0,01 a 0,50 & 38 & 0,16 & 5,4 & 4,0 & 57 & 0,16 & 5,5 & 4,0 \\
\hline 0,51 a 1,00 & 7 & 0,73 & 17,4 & 5,0 & 5 & 0,67 & 15,6 & 5,0 \\
\hline 1,01 a 1,50 & 11 & 1,26 & 35,9 & 9,1 & 9 & 1,20 & 23,1 & 6,2 \\
\hline 1,51 a 2,00 & 6 & 1,85 & 80,5 & 21,0 & 2 & 1,72 & 39,0 & 10,5 \\
\hline \multirow[t]{2}{*}{$>2,00$} & 27 & 4,95 & 127,0 & 46,5 & 4 & 3,39 & 75,5 & 19,0 \\
\hline & \multicolumn{8}{|c|}{ Anticarsia gemmatalis } \\
\hline 0 & 47 & 0 & - & - & 5 & 0 & - & - \\
\hline 0,01 a 0,50 & 29 & 0,22 & 8,8 & 4,1 & 25 & 0,12 & 5,4 & 3,8 \\
\hline 0,51 a 1,00 & 3 & 0,71 & 12,3 & 4,0 & 6 & 0,77 & 17,0 & 4,8 \\
\hline 1,01 a 1,50 & 4 & 1,31 & 31,8 & 8,3 & 7 & 1,18 & 38,3 & 10,3 \\
\hline 1,51 a 2,00 & 2 & 3,67 & 101,5 & 26,0 & 2 & 1,8 & 48,0 & 13,0 \\
\hline \multirow[t]{2}{*}{$>2,00$} & 15 & 6,32 & 165,9 & 77,5 & 5 & 4,89 & 100,0 & 43,4 \\
\hline & \multicolumn{8}{|c|}{ Rachiplusia nu } \\
\hline 0 & 21 & 0 & - & - & 40 & 0 & - & - \\
\hline 0,01 a 0,50 & 6 & 0,18 & 5,6 & 4,0 & 52 & 0,15 & 5,3 & 3,9 \\
\hline 0,51 a 1,00 & 14 & 0,73 & 24,1 & 6,5 & 7 & 0,69 & 15,0 & 4,6 \\
\hline 1,01 a 1,50 & 7 & 1,31 & 42,3 & 11,4 & 1 & 1,37 & 58,0 & 15,0 \\
\hline 1,51 a 2,0 & 2 & 1,75 & 68,5 & 18,0 & - & - & - & - \\
\hline$>2,00$ & 10 & 3,68 & 129,2 & 54,7 & - & - & - & - \\
\hline
\end{tabular}

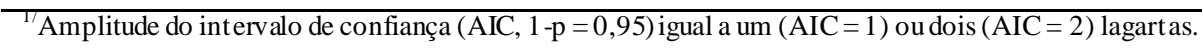

Já para coleta com rede entomológica os valores para um erro de estimação igual a dois lagartas $(\mathrm{AIC}=2)$ é respectivamente de 19 e 43,4 para $C$. includens e $A$. gemmatalis. Para a espécie $R$. nu não foi estimado valor, 
já que não foram coletadas lagartas com a rede entomológica na classe mencionada.

Como o tamanho de amostra varia com a média da densidade populacional e esta com os estágios de desenvolvimento da cultura e outros fatores ambientais, não é possível decidir por um tamanho único de amostra adequado para qualquer condição (método de coleta, cultivar e altitude da área). Em classe de densidade populacional menor de lagartas, há redução do tamanho de amostra. Desta forma, para pequenas densidades populacionais o tamanho de amostra é pequeno ou até desnecessário, pois poucos pontos amostrados seriam suficientes para decidir sobre o controle da praga.

Variações do tamanho de amostra para estimar a densidade populacional de lagartas foram relatados para a cultura da soja (Stürmer et al. 2012, Stürmer et al. 2013), cajanus (Sujithra e Chander 2016) e tomate (Cocco et al. 2015). Uma possível razão das diferenças no tamanho de amostra é a distribuição de populações.

As densidades populacionais referentes a espécie $C$. includens e R. nu e dois métodos de coleta foi maior nas lavouras de menor altitude (altitude $<150 \mathrm{~m}$ ). Por outro lado, a densidade populacional de A. gemmatalis não foi influenciada pela a altitude nos dois métodos de coleta. Esse fato poderia ser explicado, em parte, pelos fatores abióticos como a temperatura e umidade relativa do ar que poderia afetar a abundância de lagartas (Ignoffo 1992, Cespedes et al. 2008, Sujii et al. 2002).

\section{CONCLUSÕES}

O tamanho de amostra (número de pontos) para determinar a densidade populacional de lagartas varia com o método de coleta, altitude, cultivar e a densidade populacional.

Em altitudes menores que 150 m existe menor quantidade de lagartas, na maioria das cultivares (NIDERA 5909, A 8000, TIJERETA LT 2162, CODETEC 5909, NIDERA 6411) houve influencias da cultivar sobre o número de amostras.

Usando o pano-de-batida largo para a coleta de lagartas em soja, para um erro de estimação (amplitude do intervalo de confiança) igual a dois lagartas e densidade populacional na classe $>2,0$ lagartas $\mathrm{m}^{-2}$, o tamanho de amostra é 47, 78 e 18, respectivamente, para as espécies Chysodeixis includens, Anticarsia gemmatalis e R. nu.
Usando a rede entomológica para a coleta de lagartas em soja, para erro de estimação igual a dois lagartas e densidade populacional na classe $>2,0$ lagartas $\mathrm{m}^{-2}$, o tamanho de amostra é 19 e 43, respectivamente, para as espécies Chrysodeixis includens e Anticarsia gemmatalis.

\section{REFERÊNCIAS BIBLIOGRÁFICAS}

Alexandre, T. 2010. Estratégias para o manejo integrado da Pseudoplusia includens (Lepidoptera; Noctuidae, Plussinae) em soja. Tese Dr. Curitiba, Brasil, Universidade Federal de Paraná. 121 f.

Ayres, M; Ayres, J; M; Ayres, DL; Santos, AAS. 2007. BioEstat : aplicações estatísticas nas áreas das ciências biomédicas. Consultadas 23 feb 2013. Disponible en http://rs579.rapidshare.com/files/171642982/BioEstat. zip

Barbosa, JC. 2001. Métodos estatísticos aplicados à entomologia. Jaboticabal, DCE/ FCAV/UNESP. 250 p.

Céspedes, Y; del Pozo, E; García, I; Méndez, A. 2008. Efecto de la temperatura sobre el hongo entomopatógeno Nomuraea rileyi (Farlow) Samson y su efectividad sobre Spodoptera frugiperda JE Smith. Revista de Protección Vegetal 23(3):176-182.

CONAB (Companhia Nacional de Abastecimento, Brasil). 2015. $8^{\text {o }}$ Levantamento Maio 2015 In Monitoramento Agrícola: Cultivo de verão (em linha, sitio web). Consultadas 04 jun. 2015. Disponível em: http://www.conab.gov.br

Cocco, A; Serra, G; Lentini, A; Deliperi, S; Delrio, G. 2015. Spatial distribution and sequential sampling plans for Tuta absoluta (Lepidoptera: Gelechiidae) in greenhouse tomato crops. Pest Management Science 71:1311-1323.

Corrêa-Ferreira, B. 2012. Amostragem de pragas da soja. In Hoffmann-Campo, C. et al. (Eds). Soja: manejo integrado de insetos e outros artrópodes-praga. Brasilia, Brasil, EMBRAPA. p. 631-672.

Efron, B. 1979. Bootstrap method: another look at the jackknife. The Annals of Statistics 7:1-26.

Farias, P; Barbosa, JC; Busoli, AC. 2001. Distribuição espacial da lagarta-do-cartucho, Spodoptera frugiperda (J.E. Smith) (Lepidoptera: Noctuidae), na 
cultura do milho (em linha). Neotropical Entomology 30:681-689. Consultadas 04 jun. 2015. Disponível em http://dx.doi.org/10.1590/S1519-566X20 01000400025

Ferreira, DF. 2005. Estatística Básica. Lavras: UFLA. $664 \mathrm{p}$.

Franco, AA; dos Santos Queiroz, M; Peres, AR.; Rosa, ME; Campos, AR; Campos, ZR. 2014. Preferência alimentar de Anticarsia gemmatalis Hübner (Lepidoptera: Noctuidae) por cultivares de soja. Científica 42(1):32-38.

Gallo, D; Nakano, O; Silveira Neto, S; Baptista, G; Berti filho, E; Parra, J; Zucchi, R; Alves, S; Vendramim, J Marchini, L; Lopes, J; Omoto, C. 2002. Entomologia Agrícola. Piracicaba, Brasil, FEALQ. 920 p.

Guedes, J; Stecca, C; Rodrigues, R; Bigolin, M. 2010. Nova dinámica (em linha, site web). Brasil, Revista Cultivar, Consultada 06 ago 2015. Disponível em http://www.grupocultivar.com.br/

Gutierrez, M. 2009. Interações inseto-planta. In: Panizzi, A.; Parra, J. (Eds). Bioecologia e nutrição de insetos: bases para o manejo integrado de pragas. Brasília, Brasil, EMBRAPA . f. 211-249.

Ignoffo, CM. 1992. Environmental factors affecting persistence of entomopathogens (em linha). Florida Entomologist 75:516-525. Consultadas 02 ago. 2016. Disponível em <http://www.jstor.org/discover/10. 2307/3496133? uid=363288471\&uid=3737664\&uid $=2$ $129 \&$ uid $=5909624 \&$ uid $=2 \&$ uid $=70 \&$ uid $=3 \&$ uid $=67 \&$ uid=62\&uid $=23539 \&$ sid $=21101929829207>$

Lara, R; Freitas, S; Perioto, N; Paz, C. 2008. Amostragem, diversidade e sazonalidade de Hemerobiidae (Neuroptera) em Coffea arábica L. cv. Obatã (Rubiaceae). Revista Brasileira de Entomología 52(1):117-123.

Panizzi, A; Corrêa-Ferreira, B. 1978. Comparação de dois métodos de amostragem de artrópodos em soja. Anais da Sociedade Entomológica do Brasil 7:60-66.
Panizzi, A; Silva, F. 2009. Insetos sugadores de sementes (Heteroptera). In Panizzi, A; Parra, J. (eds). Bioecologia e nutrição de insetos: bases para o manejo integrado de pragas. Brasília, Brasil, EMBRAPA. f. 465-522.

R Development Core Team. 2014 R: a language and environment for statistical computing. Vienna, R Foundation for Statistical Computing. Consultadas 04 jun. 2015 Disponível em http://www.R-project.org

Storck, L; Fiorin, RA; Cargnelutti Filho, A; Guedes, JVC. 2012. A sampling procedure for quantifying mites in soybeans. Experimental \& Applied Acarology, 57(2)117-126. DOI 10.1007/s 10493-012-9547-8

Stürmer, GR; Cargnelutti Filho, A; Stefanelo, L; Guedes, JV. 2012. Eficiência de métodos de amostragem de lagartas e de percevejos na cultura de soja (em linha). Ciência Rural 42:2105-2111. Consultadas 04 jun. 2015. Disponível em http://dx.doi.org/10.1590/S010384782012005000145

Stürmer, GR; Cargnelutti Filho, A; Guedes, JVC; Stefanelo, L. 2013. Tamanho de amostra para a estimação da média de lagartas na cultura de soja. Biosci. J. Uberlândia 29(1):1596-1605.

Stürmer, GR; Cargnelutti Filho, A; Guedes, JVC; Stacke, RF. 2014. Tamanho de amostra para a estimação da população de percevejos na cultura de soja. Rev. Ciênc. Agron. 45(1):155-167.

Sujii, ER; Tigano, MS; Sosa-Gomes, D. 2002. Simulação do impacto do fungo Nomuraea rileyi em populações da lagarta da soja, Anticarsia gemmatalis (em linha). Pesquisa Agropecuária Brasileira 37(11):1551-1558. Consultadas 02 ago 2016. Disponível em http://www.scielo.br/pdf/\%0D/pab/v37n11/14519.pdf

Sujithra, M; CHander, S. 2016. Distribution pattern and sequential sampling plan for spotted pod borer, Maruca vitrata (Fabricius) (Lepidoptera: Crambidae) on pigeon pea, Cajanus cajan L. Internacional Journal of Pest Management 62:64-68. 